\title{
Circular RNA circ_0010283 regulates the viability and migration of oxidized low-density lipoprotein-induced vascular smooth muscle cells via an miR-370-3p/HMGB1 axis in atherosclerosis
}

\author{
PENG DING $^{1}$, YI DING ${ }^{2}$, YE TIAN $^{3}$ and XIAOCHUN LEI ${ }^{1}$ \\ Departments of ${ }^{1}$ Cardiovascular Medicine, ${ }^{2}$ Nephrology and Endocrinology and ${ }^{3}$ Neurology, \\ The Second Affiliated Hospital of Xi'an Medical University, Xi'an, Shanxi 710000, P.R. China
}

Received December 17, 2019; Accepted July 6, 2020

DOI: $10.3892 / \mathrm{ijmm} .2020 .4703$

\begin{abstract}
Atherosclerosis is a disease during which the inside of an artery narrows due to the accumulation of plaque, and vascular smooth muscle cells (VSMCs) are involved in the progression of atherosclerosis. Circular RNAs (circRNAs) have been reported to be involved in the progression of atherosclerosis. However, the role of circ_0010283 in atherosclerosis progression remains unclear. The present study aimed to investigate the functions and the mechanism of circ_0010283 in oxidized low-density lipoprotein (ox-LDL)-induced VSMCs and to identify new potential biomarkers for the treatment of atherosclerosis. Cell viability and migration were examined by 3-(4,5-dimethylthiazol-2-yl)-2,5-diphenyltetrazolium bromide and Transwell assays. The relationship between microRNA (miR)-370-3p and circ_0010283 or high mobility group box 1 (HMGB1) was predicated by online software and confirmed by dual-luciferase reporter assay and RNA immunoprecipitation assay. The results of the present study demonstrated that the expression levels of circ_0010283 and HMGB1 were significantly upregulated in ox-LDL-induced VSMCs compared with those in VSMCs without ox-LDL induction, whereas the expression of miR-370-3p was downregulated. Knockdown of circ_0010283 suppressed VSMC viability and migration, as well as the expression of viability-associated proteins cyclin D1 and proliferating cell nuclear antigen, and migration-associated proteins matrix metalloproteinase 2 (MMP2) and MMP9 in ox-LDL-induced VSMCs compared with untreated VSMCs. In addition, miR-370-3p was demonstrated to be a target of circ_0010283 and to target HMGB1; thus, circ_0010283 regulated HMGB1 expression via miR-370-3p.
\end{abstract}

Correspondence to: Mr. Xiaochun Lei, Department of Cardiovascular Medicine, The Second Affiliated Hospital of Xi'an Medical University, 167 Fangdong Street, Baqiao, Xi'an, Shanxi 710000, P.R. China

E-mail: i2cmqn@163.com

Key words: circ_0010283, miR-370-3p, HMGB1, viability, migration
Further experiments indicated that inhibition of miR-370-3p reversed the circ_0010283 silencing-mediated inhibitory effects on VMSC viability and migration. Additionally, the miR-370-3p-mediated suppressive effects on cell viability and migration were rescued by overexpression of HMGB1. In conclusion, circ_0010283 mediated cell viability and migration via a miR-370-3p/HMGB1 axis in ox-LDL-induced VSMCs.

\section{Introduction}

Atherosclerosis, a class of vascular diseases, is the main cause of heart disease and stroke (1). Atherosclerosis induces thrombus formation by disrupting the integrity of the arterial surface (2). Vascular smooth muscle cells (VSMCs) have been reported to be involved in the remodeling of the arterial wall in atherosclerosis (3), and their viability and migration serve a vital role in the progression of atherosclerosis $(4,5)$. Thus, it is imperative to determine the pathogenesis of atherosclerosis and the roles of VSMCs in atherosclerosis.

Circular RNAs (circRNAs) are a type of single-stranded RNAs with a covalently closed continuous loop that are produced by backsplicing (6) and are resistant to exonuclease-mediated degradation (7). Numerous studies have demonstrated that circRNAs are associated with cardiac repair (8), silica-induced macrophage activation (9), pulmonary arterial hypertension (10) and atherosclerosis (11). Microarray analysis has revealed that circ_0010283 is upregulated in oxidized low-density lipoprotein (ox-LDL)-induced VSMCs (12). However, the regulatory mechanism of circ_0010283 in atherosclerosis needs to be further investigated.

MicroRNAs (miRNAs) are short ( 22 nucleotides) conserved non-coding RNAs that mediate gene expression by binding to the 3'-untranslated region (3'UTR) of target mRNAs at the post-transcriptional level (13). Emerging evidence has revealed a core position of miRNAs in the regulation of atherosclerosis progression $(14,15)$. miR-370-3p has been reported to be associated with a number of human diseases including acute pneumonia, cerebral aneurysm and tuberculosis (16-18), and a previous study has demonstrated that miR-370-3p regulates coronary atherosclerosis progression (19). However, the 
precise mechanism of miR-370-3p in regulating atherosclerosis progression remains to be studied.

High-mobility group box 1 (HMGB1) has been reported to participate in the regulation of atherosclerosis progression. Wu et al (20) demonstrated that miR-328 mitigated ox-LDL-induced endothelial cell injury by targeting HMGB1 in atherosclerosis. Moreno et al (21) reported that HMGB1 was highly expressed in atherosclerotic plaques. Therefore, HMGB1 may be a treatment target for atherosclerosis, and the regulatory mechanism of HMGB1 needs to be explored.

The aim of the present study was to investigate the potential diagnostic biomarkers for atherosclerosis and to further understand the function and underlying regulatory mechanism of circ_0010283 in ox-LDL-induced VSMCs.

\section{Materials and methods}

Cell culture and treatment. Human vascular smooth muscle cells (VSMCs) were purchased from Otwo Biotech (Shenzhen), Inc. McCoy's 5A medium (Sigma-Aldrich; Merck KGaA) supplemented with $10 \%$ fetal bovine serum (FBS) (Sigma-Aldrich; Merck KGaA) was used to culture the cells in an atmosphere containing $5 \% \mathrm{CO}_{2}$ at $37^{\circ} \mathrm{C}$. Ox-LDL (Peking Union-Biology Co., Ltd.) was used to induce an aberrant lipid environment. Transfected cells were cultured in the medium with ox-LDL at concentrations ranging between 0 and $150 \mu \mathrm{g} / \mathrm{ml}$ for 24, 48 and $72 \mathrm{~h}$ (Fig. S1), and the normal control cells were cultured in medium without ox-LDL.

Cell transfection. Small interfering RNA (siRNA) against circ_0010283 (si-circ_0010283, 5'-GCAGTCATCTACAGA TCAA-3'), miR-370-3p mimic (referred to as miR-370-3p, 5'-GCCUGCUGGGGUGGAACCUGGU-3') and miR-370-3p inhibitor (anti-miR-370-3p, 5'-ACCAGGUUCCACCCCAGC AGGC-3'), as well as the corresponding negative controls (si-NC, 5'-CCAAAACCAGGCUUUGAUUGA-3'; miR-NC, 5'-UUCUCCGAACGUGUCACGUTT-3'; and anti-miR-NC, 5'-UCUACUCUUUCUAGGAGGUUGUGA-3') were obtained from Shanghai GenePharma Co., Ltd. HMGB1 overexpression pcDNA3.1-plasmid (referred to as HMGB1) and its negative control (referred to as vector) were acquired from Guangzhou RiboBio Co., Ltd. VSMCs $\left(1 \times 10^{5}\right.$ cells/well) were transfected with $100 \mathrm{nM}$ plasmids using Lipofectamine ${ }^{\circledR} 2000$ reagent (Invitrogen; Thermo Fisher Scientific, Inc.) according to the manufacturer's instructions. After 6 -h transfection at $37^{\circ} \mathrm{C}$, the medium was replaced with fresh medium with or without ox-LDL at $37^{\circ} \mathrm{C}$. The transfected cells were harvested after $48 \mathrm{~h}$ for use in subsequent experiments. The efficiency of transfection was measured by reverse transcription-quantitative (RT-q)PCR.

3-(4,5-Dimethylthiazol-2-yl)-2,5-diphenyltetrazolium bromide (MTT) assay. Briefly, transfected and $100 \mu \mathrm{g} / \mathrm{ml}$ ox-LDL-treated VSMCs were seeded into 96 -well plates $\left(1 \times 10^{5}\right.$ cells/well $)$ and incubated for 24, 48 or $72 \mathrm{~h}$. Subsequently, $20 \mu \mathrm{l}$ MTT solution ( $5 \mathrm{mg} / \mathrm{ml}$; Sigma-Aldrich; Merck KGaA) was added to the well to incubate at $37^{\circ} \mathrm{C}$ for $4 \mathrm{~h}$; then, the medium was discarded, and $200 \mu$ l dimethyl sulfoxide (Sigma-Aldrich; Merck KGaA) was added to the well. The optical density values were determined at $490 \mathrm{~nm}$ using the microplate reader (Bio-Rad Laboratories, Inc.).
$R N A$ isolation, $R T-q P C R$ and RNase $R$ treatment. Total RNA was isolated using TRIzol ${ }^{\circledR}$ reagent (Sigma-Aldrich; Merck $\mathrm{KGaA}$ ) and reverse-transcribed to complementary DNA using a PrimeScript RT Master Mix kit (Takara Biotechnology Co., Ltd.) according to the manufacturer's instructions. The qPCR was performed using a SYBR ${ }^{\circledR}$ Green PCR Master Mix (Vazyme Biotech Co., Ltd.), and the data were analyzed using the $2^{-\Delta \Delta \mathrm{Cq}}$ method (22). U6 was used as the control of miR-370-3p, and circ_0010283 and HMGB1 expression was normalized to $\beta$-actin. The primers used in this study were as follows: circ_0010283 forward, 5'-GAGGTGATGAAC CACCCTGG-3' and reverse, 5'-CTGAGCTGGCTGTAACCA CA-3'; linear mRNA ubiquitin protein ligase E3 component n-recognin 4 (UBR4) forward, 5'-GGTGTTCCAGAGGCT AGTGATC-3' and reverse, 5'-CCAACTGCTTCTGCGGTT CCTT-3'; miR-370-3p forward, 5'-TGTAACCAGAGAGCG GGATGT-3' and reverse, 5'-TTTTGGCATAACTAAGGC CGAA-3'; HMGB1 forward, 5'-GCGGACAAGGCCCGT TA-3' and reverse, 5'-AGAGGAAGAAGGCCGAAGGAA-3'; $\beta$-actin forward, 5'-GCACCACACCTTCTACAATG-3' and reverse, 5'-TGCTTGCTGATCCACATCTG-3'; U6 forward, 5'-TCCGGGTGATGCTTTTCCTAG-3' and reverse, 5'-CGC TTCACGAATTTGCGTGTCAT-3'; GAPDH forward, 5'-GAG TCAACGGATTTGGTCGT-3' and reverse, 5'-TTGATTTTG GAGGGATCTCG-3'.

Purified RNAs were treated with RNase R (Vazyme Biotech Co., Ltd.) for subsequent experiments. circRNA stability was assessed by RNase R experiment. The extracted RNAs from VSMCs was separated into two parts; one part was treated with RNase R digestion, whereas the other part (control) was digested with digestion buffer. The expression level of circ_0010283 and linear UBR4 mRNA were measured by RT-qPCR.

Nuclear-cytoplasmic fractionation. Cytoplasmic and nuclear RNAs were isolated using the NE-PER ${ }^{\mathrm{TM}}$ Nuclear and Cytoplasmic Extraction reagents (Thermo Fisher Scientific, Inc.) according to the manufacturer's protocol as previously described (23). Briefly, following washing with PBS, $100 \mu \mathrm{g} / \mathrm{ml}$ ox-LDL-treated VSMCs were centrifuged at $4^{\circ} \mathrm{C}$ to separate the supernatant containing cytoplasm from the nuclear components. Subsequently, the cytoplasmic and nuclear fractions were mixed with the lysis/binding solution and ethanol, respectively. The RNAs of these two fractions were obtained with an eluting solution. RT-qPCR was used to examine circ_0010283 expression in the cell cytoplasm and nucleus.

Western blotting. Proteins from VSMCs with or without ox-LDL treatment were isolated using RIPA buffer (Vazyme Biotech Co., Ltd.), and the protein concentration was determined by Detergent Compatible Bradford Protein Quantification kit (Vazyme Biotech Co., Ltd.). Equal amounts of protein ( $20 \mu \mathrm{g} /$ lane) were separated by $10 \%$ sodium dodecyl sulfate polyacrylamide gel electrophoresis (SDS-PAGE) and subsequently transferred to polyvinylidene difluoride (PVDF) membranes (Vazyme Biotech Co., Ltd.), followed by blocking with $5 \%$ skimmed milk (Vazyme Biotech Co., Ltd.) for $2 \mathrm{~h}$ at $37^{\circ} \mathrm{C}$ and washing with phosphate-buffered saline (PBS). Subsequently, the membranes were incubated with 
the following primary antibodies: Anti-cyclin D1 $(1: 1,000$; cat. no. ab16663; Abcam), anti-proliferating cell nuclear antigen (PCNA; 1:2,000; cat. no. ab18197; Abcam), anti-matrix metalloproteinase 2 (MMP2; 1:3,000; cat. no. ab97779; Abcam), anti-MMP9 (1:1,000; cat. no. ab38898; Abcam) and anti-HMGB1 (1:1,000; cat. no. ab18256; Abcam) or anti-glyceraldehyde 3-phosphate dehydrogenase (GAPDH; 1:2,500; cat. no. ab9485; Abcam) overnight at $4^{\circ} \mathrm{C}$. Following washing with PBS, the membranes were incubated with a horseradish peroxidase-conjugated anti-rabbit IgG secondary antibody (1:3,000; cat. no. ab205718; Abcam) for $3 \mathrm{~h}$ at $37^{\circ} \mathrm{C}$. The membranes were analyzed using the ChemiDoc ${ }^{\mathrm{TM}} \mathrm{MP}$ Imaging System (Bio-Rad Laboratories, Inc.) following treatment with an ECL kit (Vazyme Biotech Co., Ltd.).

Transwell assay. Transwell chambers (Beijing Solarbio Science \& Technology Co., Ltd.) were used to determine the cell migratory capacity. Transfected cells $\left(1 \times 10^{5}\right.$ cells/well $)$ were seeded into the upper chamber, and medium containing $10 \%$ FBS was placed in the lower chamber. Following treatment with crystal violet for $10 \mathrm{~min}$ at $37^{\circ} \mathrm{C}$ (Beijing Solarbio Science \& Technology Co., Ltd.), the cells were analyzed under an inverted light microscope (MTX Lab Systems, Inc.) in five random fields of view at X100 magnification.

Dual-luciferase reporter assay. The potential complementary sequences of miR-370-3p and circ_0010283 or HMGB1 were predicted using starBase (24). The wild-type (WT) sequences of circ_0010283 or HMGB1 3'UTR harboring the target sites of miR-370-3p were inserted into pGL3 vectors (Promega Corporation) to generate the luciferase reporter vectors circ_0010283 WT or HMGB1 3'UTR WT. Analogously, circ_0010283 mutant (MUT) and HMGB1 3'UTR MUT reporter vectors were established by mutating the binding sites of miR-370-3p. The vectors were co-transfected with miR-370-3p or miR-NC into VSMCs using Lipofectamine ${ }^{\circledR}$ 2000. The Dual-Glo Luciferase Assay System kit (Promega Corporation) was used to determine the relative luciferase activity. Firefly luciferase activity was normalized to Renilla luciferase.

RNA immunoprecipitation (RIP) assay. RIP was performed using a Magna RIP RNA-Binding Protein Immunoprecipitation kit (EMD Millipore) according to the manufacturer's instructions. Briefly, ox-LDL treated VSMCs were first washed with PBS. Then, the cells were centrifuged at $1,000 \times \mathrm{g}$ for $5 \mathrm{~min}$ at $4^{\circ} \mathrm{C}$, lysed with RIP buffer $(200 \mu \mathrm{l})$ and incubated with magnetic beads conjugated with an anti-argonaute 2 (anti-Ago2) antibody (EMD Millipore), and an anti-immunoglobulin G (anti-IgG) antibody (EMD Millipore) was used as the negative control. The protein was removed by Proteinase $\mathrm{K}$. The immunoprecipitated RNA was purified and analyzed by RT-qPCR.

Statistical analysis. Experimental data were calculated using GraphPad Prism 7 (GraphPad Software, Inc.) and presented as the mean \pm standard deviation. Student's t-test was used to analyze the differences between two independent groups. For multiple groups, one-way analysis of variance with Tukey's post hoc test was used to evaluate the differences. All experiments were repeated independently at least three times. $\mathrm{P}<0.05$ was considered to indicate a statistically significant difference.

\section{Results}

circ_0010283 is induced by ox-LDL and contributes to the viability and migration of ox-LDL-induced VSMCs. Ox-LDL has been reported to regulate the viability of VSMCs (25). In the present study, VSMCs were first treated with ox-LDL at concentrations ranging from 0 to $150 \mu \mathrm{g} / \mathrm{ml}$ for 24,48 and $72 \mathrm{~h}$. The results demonstrated that the viability of VSMCs was gradually induced with the increasing concentration of ox-LDL between 0 and $100 \mu \mathrm{g} / \mathrm{ml}$ and treatment time (Fig. S1). Therefore, VSMCs were treated with $100 \mu \mathrm{g} / \mathrm{ml}$ ox-LDL for 48 or $72 \mathrm{~h}$, or treated with 75 or $100 \mu \mathrm{g} / \mathrm{ml}$ ox-LDL for $72 \mathrm{~h}$, and the level of circ_0010283 was measured. As demonstrated in Fig. 1A and B, the expression of circ_0010283 was significantly increased by ox-LDL at different time points (48 and $72 \mathrm{~h}$ ) and concentrations (75 and $100 \mu \mathrm{g} / \mathrm{ml}$ ) in VSMCs compared with untreated cells. In addition, circ_0010283 was mainly distributed in the cytoplasm (Fig. 1C). Compared with linear UBR4 mRNA, circ_0010283 was more resistant to RNase R (Fig. 1D). These results suggested that circ_0010283 may function in atherosclerosis progression. Thus, the role of circ_0010283 in ox-LDL-induced VSMCs was explored. VSMCs were treated with ox-LDL, ox-LDL + si-NC or ox-LDL + si-circ_0010283, and the knockdown efficiency of si-circ_0010283 in VSMCs treated with ox-LDL or without treatment was verified by RT-qPCR (Figs. 1E and S2A). MTT assay results demonstrated that downregulation of circ_0010283 notably suppressed ox-LDL-induced viability in VSMCs (Fig. 1F). In addition, circ_0010283 silencing decreased the levels of the viability-associated proteins cyclin D1 and PCNA in ox-LDL-induced VSMCs compared with those in the ox-LDL + si-NC group (Fig. 1G). The migration of ox-LDL-induced VSMCs was also repressed by circ_0010283 knockdown compared with that of VMSCs transfected with si-NC (Fig. 1H). Additionally, ox-LDL-induced MMP2 and MMP9 levels were downregulated by si-circ_0010283 in VSMCs treated with ox-LDL (Fig. 1I) Therefore, the results of the present study suggested that circ_0010283 may serve a promoting role in the viability and migration of ox-LDL-induced VSMCs.

circ_0010283 regulates cell viability and migration by targeting miR-370-3p in ox-LDL-induced VSMCs. circRNAs have been reported to regulate a number of human diseases by interacting with miRNAs $(26,27)$. The potential miRNA targets for circ_0010283 were screened in the present study using the starBase database, and the results demonstrated that miR-370-3p harbored the binding sites of circ_0010283 (Fig. 2A). To verify the interaction between circ_0010283 and miR-370-3p, dual-luciferase and RIP assays were performed. The dual-luciferase reporter assay results revealed that miR-370-3p significantly diminished the luciferase activity of circ_0010283 WT, but not that of circ_0010283 MUT (Fig. 2B). The results of the RIP assay demonstrated that the relative enrichment of circ_0010283 and miR-370-3p was higher in the anti-Ago2 group compared with that in anti-IgG group (Fig. 2C). In addition, the expression levels of miR-370-3p were downregulated 
A

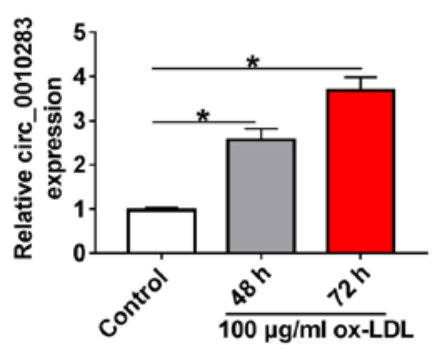

D

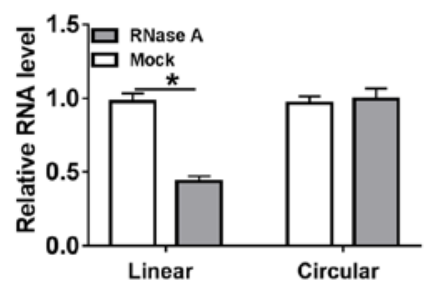

G

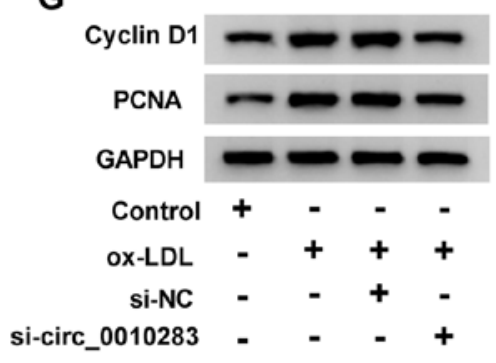

H
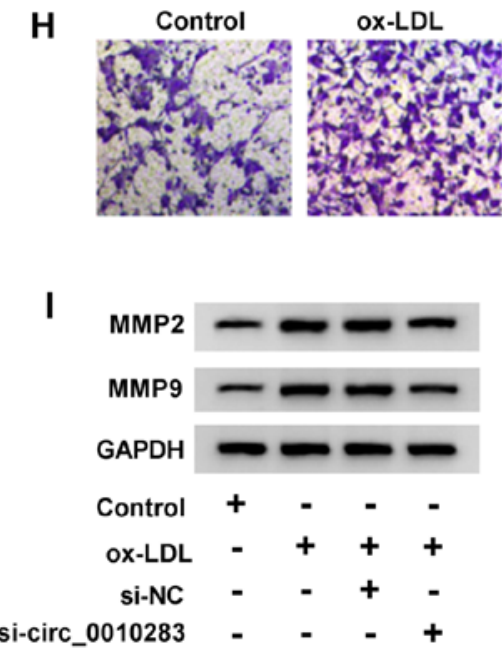



E
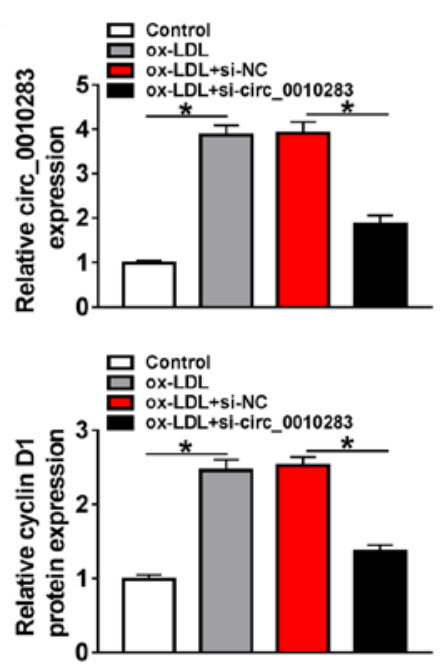

$\mathbf{F}$
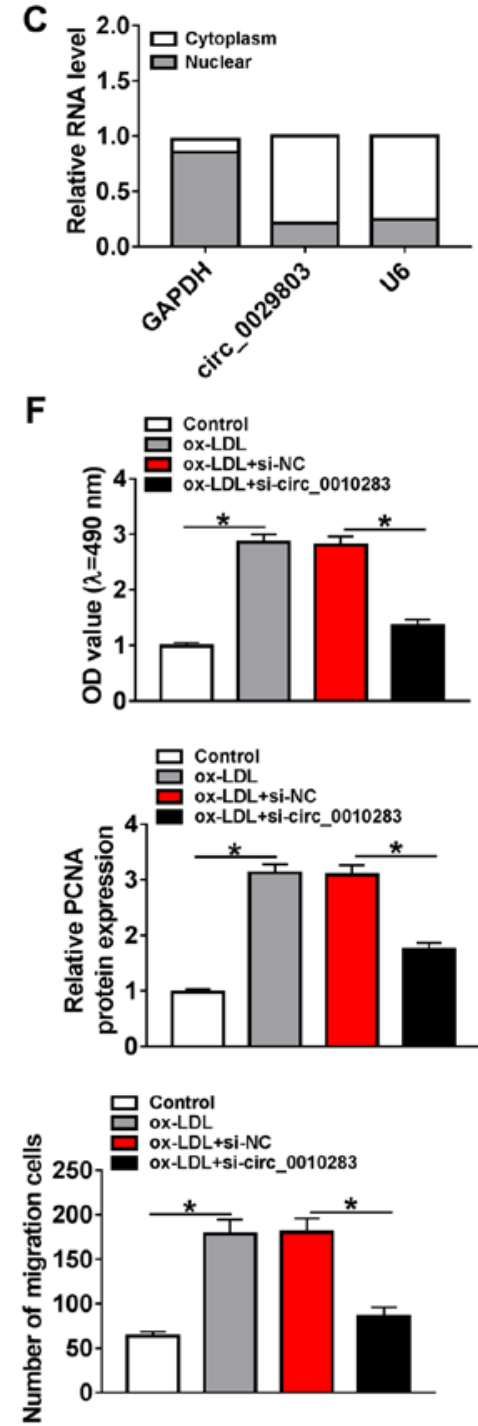

ox-LDL+
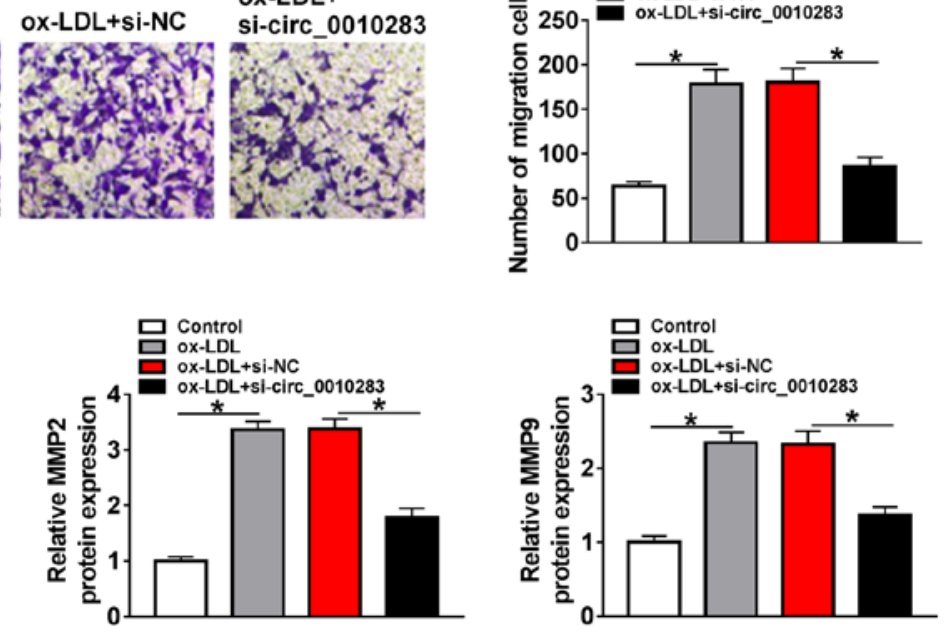

Figure 1. circ_0010283 expression is induced by ox-LDL and facilitates the viability and migration of ox-LDL-induced VSMCs. (A) The expression levels of circ_0010283 in VSMCs treated with $100 \mu \mathrm{g} / \mathrm{ml}$ ox-LDL or for 48 or $72 \mathrm{~h}$ and untreated cells were determined by RT-qPCR. (B) The expression levels of circ_0010283 in VSMCs treated with 75 or $100 \mu \mathrm{g} / \mathrm{ml}$ ox-LDL for $72 \mathrm{~h}$ was detected by RT-qPCR. (C) The expression levels of circ_0010283 in the nucleus and the cytoplasm were detected by RT-qPCR. GAPDH and U6 were used as positive controls in the nucleus and cytoplasm, respectively. (D) The relative expression levels of circ_0010283 in VSMCs treated with RNase R or not were measured by RT-qPCR. (E) The levels of circ_0010283 in VSMCs treated with $100 \mu \mathrm{g} / 1$ ox-LDL or ox-LDL + si-circ_0010283 and the corresponding controls was determined by RT-qPCR. (F) The viability of VSMCs treated with $100 \mu \mathrm{g} / \mathrm{ml}$ ox-LDL or ox-LDL + si-circ_0010283 and the corresponding controls was determined by MTT assay. (G) The protein levels of cyclin D1 and PCNA were detected by western blotting in VSMCs treated with $100 \mu \mathrm{g} / \mathrm{ml}$ ox-LDL or ox-LDL + si-circ_0010283 and the corresponding controls. (H) The migration of VSMCs treated with $100 \mu \mathrm{g} / \mathrm{ml}$ ox-LDL or ox-LDL + si-circ_0010283 and the corresponding controls was evaluated by Transwell assay. (I) The protein levels of MMP2 and MMP9 were measured by western blotting in VSMCs treated with $100 \mu \mathrm{g} / \mathrm{ml}$ ox-LDL or ox-LDL + si-circ_0010283 and the corresponding controls. *P<0.05. VMSCs, vascular smooth muscle cells; ox-LDL, oxidized low-density lipoprotein; RT-qPCR, reverse transcription-quantitative PCR; si, small interfering; circ, circular RNA; NC, negative control; PCNA, proliferating cell nuclear antigen; MMP, matrix metalloproteinase; OD, optical density.

in ox-LDL-induced VSMCs compared with those in the control group (Fig. 2D and E). circ_0010283 silencing increased the expression level of miR-370-3p in ox-LDL-induced VSMCs compared with that in the group transfected with si-NC alone
(Fig. 2F). Subsequently, the overexpression or inhibition efficiency of miR-370-3p mimic or anti-miR-370-3p in VSMCs was verified by RT-qPCR (Fig. S2B and C). VSMCs were treated with ox-LDL, ox-LDL + si-circ_0010283 or ox-LDL + 
A





C



$\mathbf{F}$
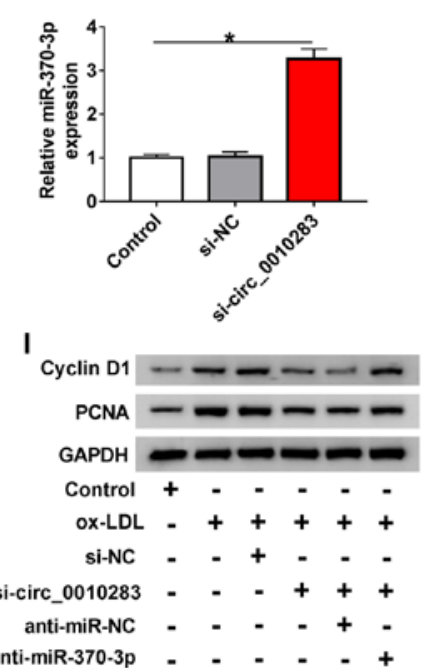

anti-miR-370-3p - - - - - +



G

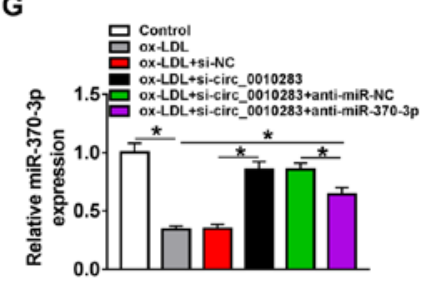

E

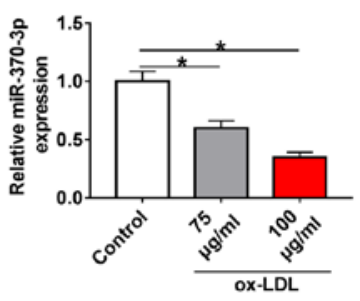

H 吕 control



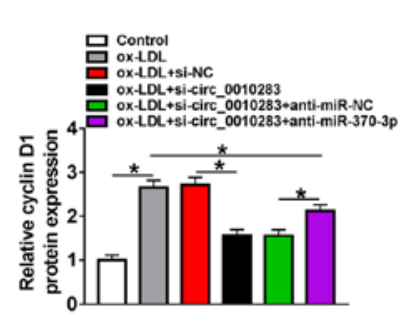

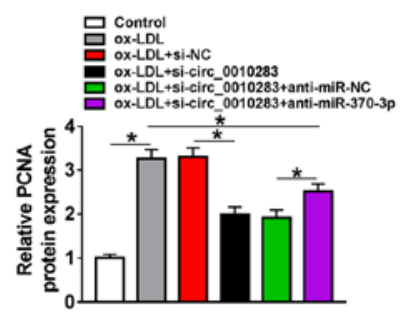

J

Control Ox-LDL $\quad$ Ox-LDL+SI-NC ox-LDL+

ox-LDL+

ox-LDL+ si-circ_0010

si-circ_0010283+

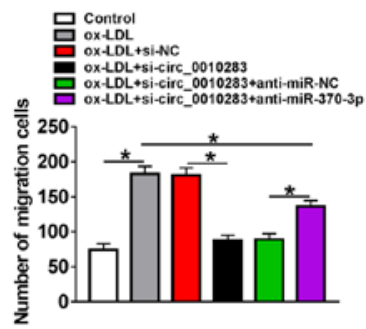

$\mathbf{K}$
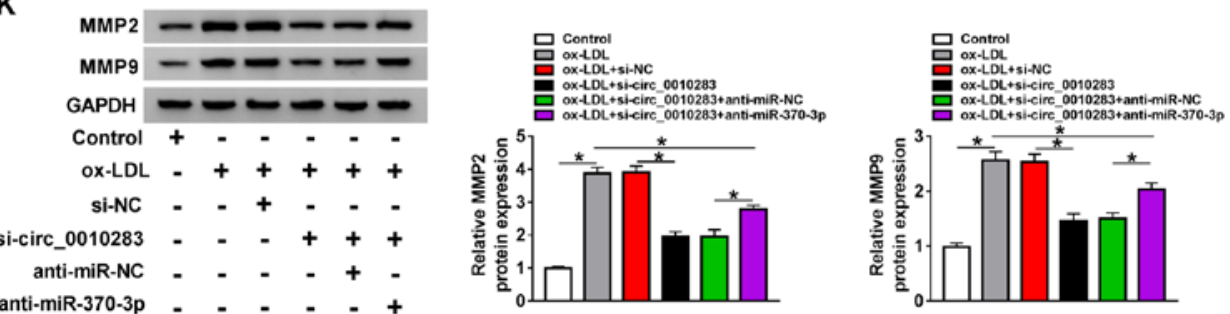

Figure 2. circ_0010283 regulates cell viability and migration by targeting miR-370-3p in ox-LDL-induced VSMCs. (A) The putative binding sites between circ_0010283 and miR-370-3p were predicted by starBase. (B) The luciferase activity of circ_0010283 WT and circ_0010283 MUT was detected in VSMCs co-transfected with miR-370-3p or miR-NC. (C) The RIP assay was performed to determine the interaction between circ_0010283 and miR-370-3p, and anti-IgG was used as the control. (D) The expression of miR-370-3p in VSMCs treated with 0 or $100 \mu \mathrm{g} / \mathrm{ml}$ ox-LDL for 48 or $72 \mathrm{~h}$ was assessed by RT-qPCR. (E) The level of miR-370-3p in VSMCs treated with 75 or $100 \mu \mathrm{g} / \mathrm{ml}$ ox-LDL for $72 \mathrm{~h}$ was measured by RT-qPCR. (F) The expression of miR-370-3p in VSMCs transfected with si-circ_0010283 or si-NC was determined by RT-qPCR. (G) The levels of miR-370-3p in VSMCs treated with $100 \mu \mathrm{g} / \mathrm{ml}$ ox-LDL, ox-LDL + si-circ_0010283 or ox-LDL + si-circ_0010283 + anti-miR-370-3p as well as the corresponding controls were assessed by RT-qPCR. (H) The viability of VSMCs treated with $100 \mu \mathrm{g} / \mathrm{ml}$ ox-LDL, ox-LDL + si-circ_0010283 or ox-LDL + si-circ_0010283 + anti-miR-370-3p as well as the corresponding controls was determined by an MTT assay. (I) The protein levels of cyclin D1 and PCNA in VSMCs treated with $100 \mu \mathrm{g} / \mathrm{ml}$ ox-LDL, ox-LDL + si-circ_0010283 or ox-LDL + si-circ_0010283 + anti-miR-370-3p as well as the corresponding controls were determined by western blotting. (J) The migratory ability of VSMCs treated with $100 \mu \mathrm{g} / \mathrm{ml}$ ox-LDL, ox-LDL + si-circ_0010283 or ox-LDL + si-circ_0010283 + anti-miR-370-3p as well as the corresponding controls was assessed by Transwell assay. (K) The protein levels of MMP2 and MMP9 in VSMCs treated with $100 \mu \mathrm{g} / \mathrm{ml}$ ox-LDL, ox-LDL + si-circ_0010283 or ox-LDL + si-circ_0010283 + anti-miR-370-3p as well as the corresponding controls were detected by western blotting. "P<0.05. VMSCs, vascular smooth muscle cells; ox-LDL, oxidized low-density lipoprotein; RT-qPCR, reverse transcription-quantitative PCR; si, small interfering; circ, circular RNA; NC, negative control; miR, microRNA; WT, wild-type; MUT, mutant; RIP, RNA immunoprecipitation; Ago2, argonaute 2; IgG, immunoglobulin G; OD, optical density. 
si-circ_0010283 + anti-miR-370-3p to investigate the interaction between circ_0010283 and miR-370-3p. The RT-qPCR results demonstrated that circ_0010283 silencing-induced miR-370-3p level was reduced by anti-miR-370-3p in ox-LDL-induced VSMCs (Fig. 2G). In addition, anti-miR-370-3p rescued the si-circ_0010283-suppressed viability of ox-LDL-induced VSMCs (Fig. 2H and I). The circ_0010283 knockdown-mediated inhibitory effect on cell migration was also partly reversed by anti-miR-370-3p. As demonstrated by the migratory rates and MMP2 and MMP9 levels, co-transfection with si-circ_0010283 + anti-miR-370-3p partially restored the suppressive effects of si-circ_0010283 on the migratory rate and the expression of MMP2 and MMP9 in ox-LDL-induced VSMCs (Fig. 2J and K). These results suggested that circ_0010283 affected the viability and migration of ox-LDL-induced VSMCs by targeting miR-370-3p.

circ_0010283 regulates HMGB1 expression via miR-370-3p. To further determine the regulatory mechanism of miR-370-3p, its possible target genes were predicted by starBase, which identified that miR-370-3p could bind to the 3'UTR of HMGB1 (Fig. 3A). This interaction was confirmed by the dual-luciferase reporter assay (Fig. 3B). The mRNA and protein levels of HMGB1 were also measured, and the results demonstrated that HMGB1 was upregulated in VSMCs treated with $100 \mu \mathrm{g} / \mathrm{ml}$ ox-LDL for 48 or $72 \mathrm{~h}$ (Fig. 3C and D). Analogously, the expression level of HMGB1 was significantly increased in VSMCs treated with 75 or $100 \mu \mathrm{g} / \mathrm{ml}$ ox-LDL for $72 \mathrm{~h}$ (Fig. 3E and F). In addition, miR-370-3p mimics decreased the mRNA and protein levels of HMGB1 (Fig. 3G and H). Knockdown of circ_0010283 downregulated HMGB1 expression, whereas anti-miR-370-3p reversed this effect (Fig. 3I and J). These results suggested that HMGB1 was a target of miR-370-3p, and circ_0010283 positively regulated HMGB1 expression via miR-370-3p.

Overexpression of $H M G B 1$ reverses the miR-370-3p-mediated suppression on the viability and migration of ox- $L D L$-induced $V S M C s$. To investigate the regulatory relationship between miR-370-3p and HMGB1 in atherosclerosis progression, VSMCs were transfected with an HMGB1 overexpression vector, and the transfection efficiency was verified in ox-LDL-induced and untreated VSMCs by RT-qPCR and western blotting (Figs. S3A, B, S2D and E). Subsequently, the mRNA and protein levels of HMGB1 in VSMCs treated with ox-LDL, ox-LDL + miR-370-3p or ox-LDL + miR-370-3p + HMGB1, as well as matched controls were detected. The results demonstrated that HMGB1 expression was downregulated in ox-LDL-induced VSMCs treated with the miR-370-3p mimic compared with those treated with ox-LDL and miR-NC; however, the expression of HMGB1 was upregulated in the ox-LDL + miR-370-3p group following transfection with the HMGB1 overexpression vector (Fig. 4A and B). The results of the MTT assay revealed that overexpression of HMGB1 significantly inhibited the miR-370-3p-mediated repression of the viability of ox-LDL-induced VSMCs (Fig. 4C). In addition, the decreased protein levels of cyclin D1 and PCNA in the ox-LDL + miR-370-3p group were partially reversed following HMGB1 overexpression (Fig. 4D). Analogously, the miR-370-3p-mediated inhibitory effect on the migration of ox-LDL-induced VSMCs was reversed by HMGB1 overexpression (Fig. 4E). In addition, the low protein levels of MMP2 and MMP9 in ox-LDL-induced VSMCs transfected with the miR-370-3p mimics were rescued following HMGB1 overexpression (Fig. 4F). In summary, these results suggested that $\mathrm{miR}-370-3 \mathrm{p}$ regulated the viability and migration of ox-LDL-induced VSMCs by targeting HMGB1.

\section{Discussion}

Atherosclerosis is a growing threat to human health, and the dysfunction of VSMCs has been reported to be involved in the formation of atherosclerosis $(28,29)$. In the present study, VSMCs were treated with ox-LDL to establish the cell model for atherosclerosis based on previous studies $(29,30)$. Recently, circRNAs have been identified to serve a pivotal role in various human diseases. Garikipati et al (8) have reported that circular RNA circFndc3b regulate cardiac repair after myocardial infarction. Zhou et al (9) have demonstrated that circRNA HECT domain-containing E3 ubiquitin protein ligase 1 (HECTD1) mediates silica-induced macrophage activation by regulating the circHECTD1/HECTD1 axis and zinc finger CCCH-type containing 12A-dependent ubiquitination process. Zhou et al (10) have reported that circular RNA hsa_circ_0016070 is associated with pulmonary arterial hypertension. In addition, circRNA antisense non-coding RNA in the INK4 locus and circRNA checkpoint with forkhead and ring finger domains (circCHFR) have been confirmed to modulate the progression of atherosclerosis $(11,12)$. In the present study, the expression of circ_0010283 was elevated in ox-LDL-induced VSMCs compared with that in non-induced cells, which was also supported by the microarray results of a previous study (12). In addition, the results of the present study demonstrated that circ_0010283 was induced by ox-LDL in a time- and concentration-dependent manner in VSMCs, suggesting that circ_0010283 may be associated with the progression of atherosclerosis. A previous study has demonstrated that circCHFR, which is also induced by ox-LDL in VSMCs, promotes the development of atherosclerosis (31). Similarly, the present results indicated that knockdown of circ_0010283 hindered the viability and migration of ox-LDL-induced VSMCs, and reduced the expression levels of the viability-associated proteins cyclin D1 and PCNA, and the migration-associated proteins MMP2 and MMP9. Taken together, these results suggested that circ_0010283 contributed to atherosclerosis progression and may be a potential biomarker for atherosclerosis.

Emerging evidence has revealed that circRNAs act as miRNA sponges in various human diseases, including diabetic retinal vascular dysfunction and myocardial fibrosis $(26,27)$. The results of the present study demonstrated that miR-370-3p was a target of circ_0010283. miR-370-3p has been reported to partake in a number of human diseases. For example, Zhang et al (16) have demonstrated that miR-370-3p is associated with acute pneumonia, and Hou et al (17) have reported that miR-370-3p suppresses VSMC viability in cerebral aneurysm. In addition, circ_0003204 can directly target miR-370-3p to repress the progression of atherosclerosis (32). In the present study, miR-370-3p was downregulated in ox-LDL-induced VSMCs compared with non-induced cells, and was negatively 
A

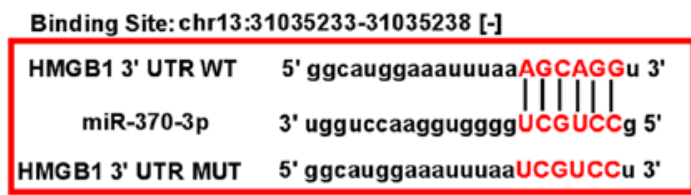

C


I



$\mathbf{J}$
B



$E$
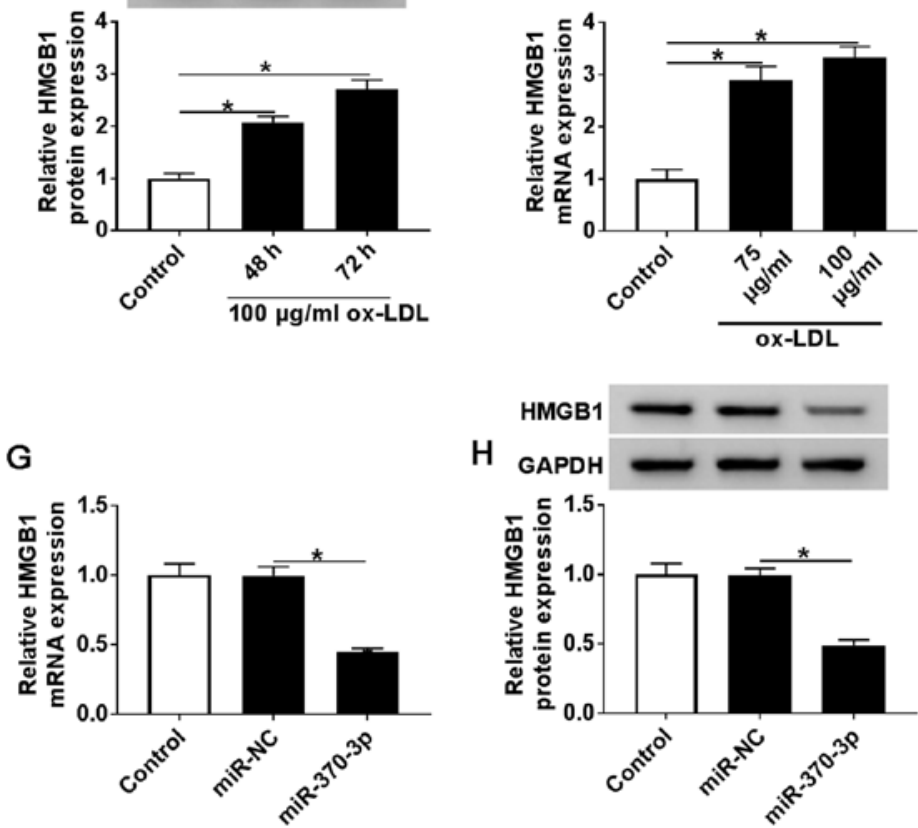


Figure 3. circ_0010283 regulates HMGB1 expression via miR-370-3p. (A) The potential target sites between miR-370-3p and HMGB1 were predicted by starBase. (B) The luciferase activity of HMGB1 3'UTR WT and HMGB1 3'UTR MUT was detected in VSMCs co-transfected with miR-370-3p or miR-NC (C and D) The mRNA and protein levels of HMGB1 in VSMCs treated with 0 or $100 \mu \mathrm{g} / \mathrm{ml}$ ox-LDL for 48 or $72 \mathrm{~h}$ were determined by RT-qPCR and western blotting, respectively. (E and F) The mRNA and protein levels of HMGB1 in VSMCs treated with 75 or $100 \mu \mathrm{g} / \mathrm{ml}$ ox-LDL for $72 \mathrm{~h}$ were assessed by RT-qPCR and western blotting, respectively. (G and H) The mRNA and protein levels of HMGB1 in VSMCs transfected with the miR-370-3p mimics or NC were detected by RT-qPCR and western blotting, respectively. (I and J) The mRNA and protein levels of HMGB1 in VSMCs treated with si-circ_0010283 or si-circ_0010283 + anti-miR-370-3p as well as the corresponding controls were determined by RT-qPCR and western blotting, respectively. "P<0.05. VMSCs, vascular smooth muscle cells; ox-LDL, oxidized low-density lipoprotein; RT-qPCR, reverse transcription-quantitative PCR; si, small interfering; circ, circular RNA; NC, negative control; miR, microRNA; UTR, untranslated region; WT, wild-type; MUT, mutant; HMGB1, high mobility group box 1.

modulated by circ_0010283. In-depth experiments demonstrated that inhibition of miR-370-3p reversed the circ_0010283 silencing-mediated repressive effects on the viability and migration of ox-LDL-induced VSMCs. These results suggested that circ_0010283 may regulate the progression of atherosclerosis by sponging miR-370-3p. In addition, the inhibitory role of miR-370-3p in atherosclerosis progression was observed, which was consistent with previous studies $(19,32)$.

To deeply understand the regulatory mechanism of miR-370-3p in atherosclerosis, its target genes were predicted, and HMGB1 was identified as a target of miR-370-3p. HMGB1 has been reported to accelerate foam cell formation and cholesterol accumulation in VSMCs (33). In addition, HMGB1 inhibition may be a potential therapeutic strategy for atherosclerosis (34). In the present study, the mRNA and protein levels of HMGB1 were measured, and the results demonstrated that the expression levels of HMGB1 were upregulated in ox-LDL-induced VSMCs compared with those in untreated cells, which was in line with a previous study (35). In addition, HMGB1 was demonstrated to be negatively regulated by 
A



D



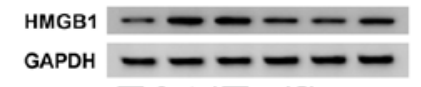

\section{B}
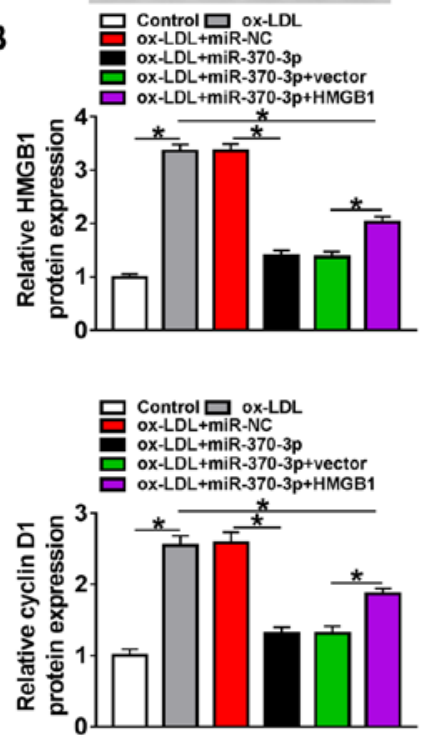

C

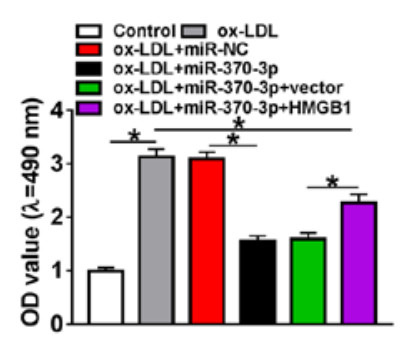

E
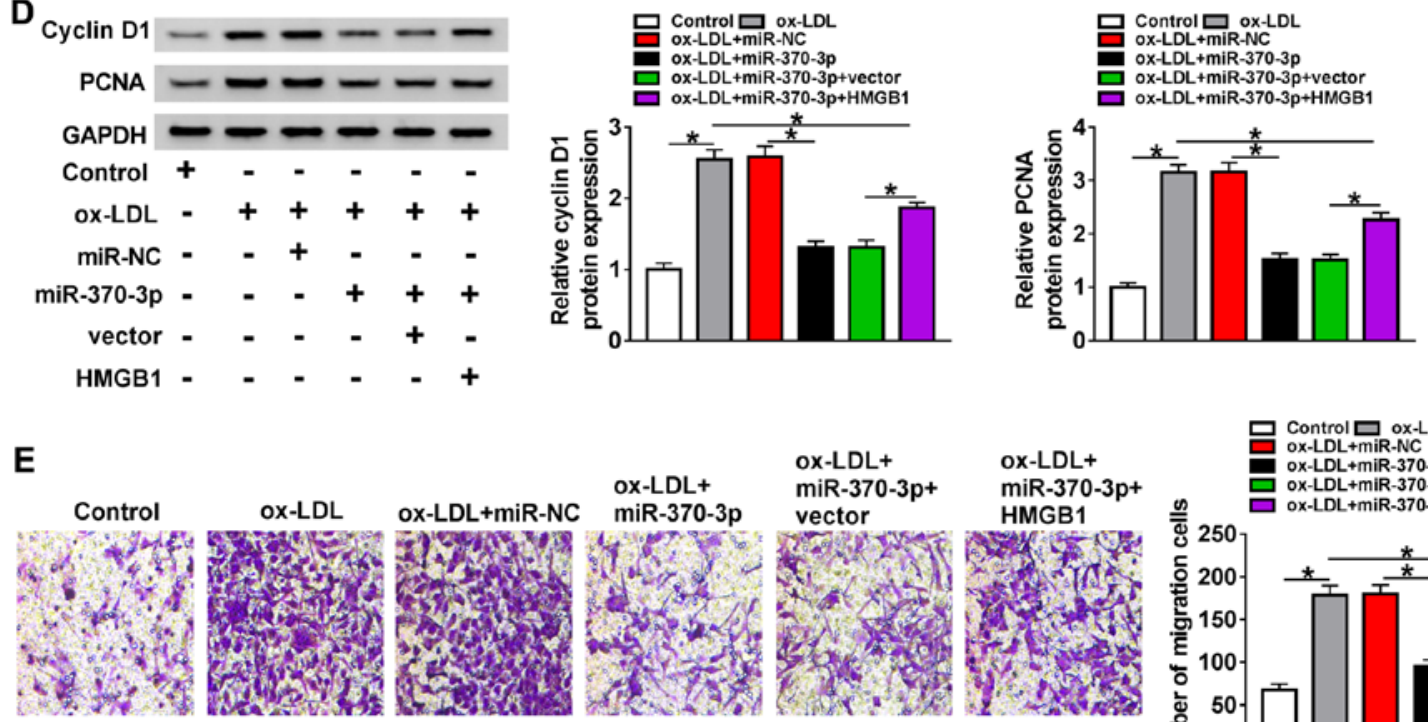

$\mathbf{F}$

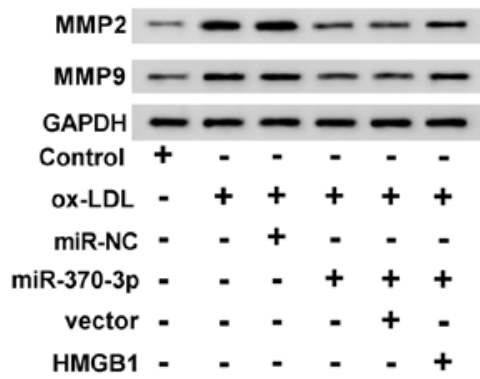



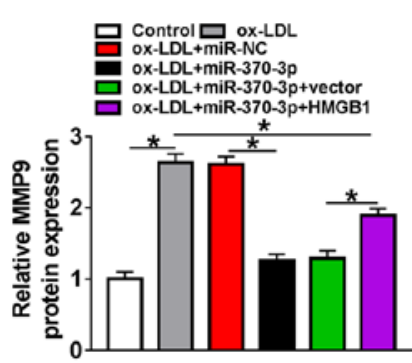

Figure 4. Overexpression of HMGB1 reverses the miR-370-3p-mediated effects on VSMC viability and migration. VSMCs were treated with $100 \mu \mathrm{g} / \mathrm{ml} \mathrm{ox}-\mathrm{LDL}$, ox-LDL + miR-370-3p or ox-LDL + miR-370-3p + HMGB1 as well as the corresponding controls. (A and B) The mRNA and protein levels of HMGB1 were assessed by reverse transcription-quantitative PCR and western blotting, respectively. (C) Cell viability was determined by MTT assay. (D) The protein levels of cyclin D1 and PCNA in all groups were detected by western blot. (E) Cell migration was assessed by Transwell assay. (F) The protein levels of MMP2 and MMP9 in the samples were measured by western blotting. "P<0.05. VMSCs, vascular smooth muscle cells; ox-LDL, oxidized low-density lipoprotein; miR, microRNA; NC, negative control; HMGB1, high mobility group box 1; PCNA, proliferating cell nuclear antigen; MMP, matrix metalloproteinase; OD, optical density.

miR-370-3p in the present study, and circ_0010283 upregulated HMGB1 expression via miR-370-3p. Further results indicated that overexpression of HMGB1 reversed the miR-370-3p-mediated suppressive effects on the viability and migration of ox-LDL-induced VSMCs. Additionally, the low mRNA and protein levels of HMGB1 in the si-circ_0010283 group were reversed following transfection with a miR-370-3p inhibitor. Taken together, these results suggested that circ_0010283 may act as a sponge of miR-370-3p to upregulate the expression of HMGB1, thus promoting the viability and migration of ox-LDL-induced VSMCs (Fig. S4).

There were certain limitations in the current study. Previous studies have elucidated that HMGB1 affects VSMC development by regulating various signaling pathways, such as PI3K/Akt and p38 mitogen-activated protein kinase/nuclear factor $\kappa \mathrm{B}$ pathways $(36,37)$. Whether the circ_0010283/miR-370-3p/HMGB1 axis regulates atherosclerosis progression via these pathways needs to be further explored. Addtionally, the role of the circ_0010283/miR-370-3p/HMGB1 axis in atherosclerosis in vivo also has not been demonstrated. These issues will be the focus of our future study.

In conclusion, the results of the present study demonstrated that circ_0010283 was significantly upregulated in ox-LDL-induced VSMCs. In addition, circ_0010283 regulated the viability and migration of ox-LDL-induced VSMCs via the miR-370-3p/HMGB1 axis. This novel mechanism 
may provide new effective therapeutic methods for atherosclerosis.

\section{Acknowledgements}

Not applicable.

\section{Funding}

No funding was received.

\section{Availability of data and materials}

The datasets analyzed and/or generated during the present study are available from the corresponding author on reasonable request.

\section{Authors' contributions}

YD and YT conceived and designed the study. YT and XL collected and analyzed the data. PD and YT performed and validated the experiments. PD, YD and YT wrote, edited and reviewed the manuscript. All authors read and approval the final manuscript.

\section{Ethics approval and consent to participate}

Not applicable.

\section{Patient consent for publication}

Not applicable.

\section{Competing interests}

The authors declare that they have no competing interests.

\section{References}

1. Lusis AJ: Atherosclerosis. Nature 407: 233-241, 2000.

2. Hansson GK, Libby P and Tabas I: Inflammation and plaque vulnerability. J Intern Med 278: 483-493, 2015.

3. Chistiakov DA, Orekhov AN and Bobryshev YV: Vascular smooth muscle cell in atherosclerosis. Acta Physiol (Oxf) 214: 33-50, 2015.

4. Tapia-Vieyra JV, Delgado-Coello B and Mas-Oliva J: Atherosclerosis and cancer; A resemblance with far-reaching implications. Arch Med Res 48: 12-26, 2017.

5. Bennett MR, Sinha S and Owens GK: Vascular smooth muscle cells in atherosclerosis. Circ Res 118: 692-702, 2016.

6. Kristensen LS, Andersen MS, Stagsted LVW, Ebbesen KK, Hansen TB and Kjems J: The biogenesis, biology and characterization of circular RNAs. Nat Rev Genet 20: 675-691, 2019.

7. Jeck WR, Sorrentino JA, Wang K, Slevin MK, Burd CE, Liu J, Marzluff WF and Sharpless NE: Circular RNAs are abundant, conserved, and associated with ALU repeats. RNA 19: 141-157, 2013

8. Garikipati VNS, Verma SK, Cheng Z, Liang D, Truongcao MM, Cimini M, Yue Y, Huang G, Wang C, Benedict C, et al: Circular RNA CircFndc $3 b$ modulates cardiac repair after myocardial infarction via FUS/VEGF-A axis. Nat Commun 10: 4317, 2019.

9. Zhou Z, Jiang R, Yang X, Guo H, Fang S, Zhang Y, Cheng Y, Wang J, Yao $\mathrm{H}$ and Chao J: circRNA mediates silica-induced macrophage activation via HECTD1/ZC3H12A-dependent ubiquitination. Theranostics 8: 575-592, 2018.

10. Zhou S, Jiang H, Li M, Wu P, Sun L, Liu Y, Zhu K, Zhang B, Sun G, Cao C and Wang R: Circular RNA hsa_circ_0016070 is associated with pulmonary arterial hypertension by promoting PASMC viability. Mol Ther Nucleic Acids 18: 275-284, 2019.
11. Holdt LM, Stahringer A, Sass K, Pichler G, Kulak NA, Wilfert W, Kohlmaier A, Herbst A, Northoff BH, Nicolaou A, et al: Circular non-coding RNA ANRIL modulates ribosomal RNA maturation and atherosclerosis in humans. Nat Commun 7: 12429, 2016.

12. Yang L, Yang F, Zhao H, Wang M and Zhang Y: Circular RNA circCHFR facilitates the proliferation and migration of vascular smooth muscle via miR-370/FOXO1/Cyclin D1 Pathway. Mol Ther Nucleic Acids 16: 434-441, 2019.

13. Gebert LFR and MacRae IJ: Regulation of microRNA function in animals. Nat Rev Mol Cell Biol 20: 21-37, 2019.

14. Schober A, Nazari-Jahantigh M, Wei Y, Bidzhekov K, Gremse F, Grommes J, Megens RT, Heyll K, Noels H, Hristov M, et al: MicroRNA-126-5p promotes endothelial proliferation and limits atherosclerosis by suppressing Dlk1. Nat Med 20: 368-376, 2014.

15. Wei Y, Nazari-Jahantigh $M$, Chan L, Zhu M, Heyll K, Corbalán-Campos J, Hartmann P, Thiemann A, Weber C and Schober A: The microRNA-342-5p fosters inflammatory macrophage activation through an Akt1- and microRNA-155-dependent pathway during atherosclerosis. Circulation 127: 1609-1619, 2013.

16. Zhang Y, Zhu Y, Gao G and Zhou Z: Knockdown XIST alleviates LPS-induced WI-38 cell apoptosis and inflammation injury via targeting miR-370-3p/TLR4 in acute pneumonia. Cell Biochem Funct 37: 348-358, 2019.

17. Hou WZ, Chen XL, Wu W and Hang CH: MicroRNA-370-3p inhibits human vascular smooth muscle cell proliferation via targeting KDR/AKT signaling pathway in cerebral aneurysm. Eur Rev Med Pharmacol Sci 21: 1080-1087, 2017.

18. Lyu L, Zhang X, Li C, Yang T, Wang J, Pan L, Jia H, Li Z, Sun Q, Yue L, et al: Small RNA profiles of serum exosomes derived from individuals with latent and active tuberculosis. Front Microbiol 10: 1174, 2019.

19. Shi $X$ and Chen $X$ : Effect of microRNA-370 on coronary atherosclerosis and its underlying mechanism. Exp Ther Med 17: 115-122, 2019.

20. Wu CY, Zhou ZF, Wang B, Ke ZP, Ge ZC and Zhang XJ: MicroRNA-328 ameliorates oxidized low-density lipoprotein-induced endothelial cells injury through targeting HMGB1 in atherosclerosis. J Cell Biochem: Oct 15, 2018 (Epub ahead of print).

21. Moreno JA, Sastre C, Madrigal-Matute J, Muñoz-García B, Ortega L, Burkly LC, Egido J, Martín-Ventura JL and Blanco-Colio LM: HMGB1 expression and secretion are increased via TWEAK-Fn14 interaction in atherosclerotic plaques and cultured monocytes. Arterioscler Thromb Vasc Biol 33: 612-620, 2013.

22. Livak KJ and Schmittgen TD: Analysis of relative gene expression data using real-time quantitative PCR and the 2(-Delta Delta C(T)) method. Methods 25: 402-408, 2001.

23. Tsai NP, Lin YL, Tsui YC and Wei LN: Dual action of epidermal growth factor: Extracellular signal-stimulated nuclear-cytoplasmic export and coordinated translation of selected messenger RNA. J Cell Biol 188: 325-333, 2010.

24. Li JH, Liu S, Zhou H, Qu LH and Yang JH: StarBase v2.0: Decoding miRNA-ceRNA, miRNA-ncRNA and protein-RNA interaction networks from large-scale CLIP-Seq data. Nucleic Acids Res 42 (Database Issue): D92-D97, 2014.

25. Hartley A, Haskard D and Khamis R: Oxidized LDL and anti-oxidized LDL antibodies in atherosclerosis-novel insights and future directions in diagnosis and therapy $<$ sup $/>$. Trends Cardiovasc Med 29: 22-26, 2019.

26. Zhu K, Hu X, Chen H, Li F, Yin N, Liu AL, Shan K, Qin YW, Huang X, Chang Q, et al: Downregulation of circRNA DMNT3B contributes to diabetic retinal vascular dysfunction through targeting miR-20b-5p and BAMBI. EBioMedicine 49: 341-353, 2019.

27. Zhou B and Yu JW: A novel identified circular RNA, circRNA_010567, promotes myocardial fibrosis via suppressing miR-141 by targeting TGF- $\beta 1$. Biochem Biophys Res Commun 487: 769-775, 2017.

28. Kattoor AJ, Kanuri SH and Mehta JL: Role of Ox-LDL and LOX-1 in atherogenesis. Curr Med Chem 26: 1693-1700, 2019.

29. Liu M, Song Y and Han Z: Study on the effect of LncRNA AK094457 on OX-LDL induced vascular smooth muscle cells. Am J Transl Res 11: 5623-5633, 2019.

30. Xu K, Xiwen Liu, Ren G, Yin D, Guo S and Zhao Y: Depletion of CPEB1 protects against oxidized LDL-induced endothelial apoptosis and inflammation though SIRT1/LOX-1 signalling pathway. Life Sci 239: 116874, 2019.

31. Zhuang JB, Li T, Hu XM, Ning M, Gao WQ, Lang YH, Zheng WF and Wei J: Circ_CHFR expedites cell growth, migration and inflammation in ox-LDL-treated human vascular smooth muscle cells via the miR-214-3p/Wnt $3 / \beta$-catenin pathway. Eur Rev Med Pharmacol Sci 24: 3282-3292, 2020. 
32. Zhang S, Song G, Yuan J, Qiao S, Xu S, Si Z, Yang Y, Xu X and Wang A: Circular RNA circ_0003204 inhibits proliferation, migration and tube formation of endothelial cell in atherosclerosis via miR-370-3p/TGF $3 R$ 2/phosph-SMAD3 axis. J Biomed Sci 27: 11, 2020

33. Wang R, Wu W, Li W, Huang S, Li Z, Liu R, Shan Z, Zhang C, $\mathrm{Li} \mathrm{W}$ and Wang S: Activation of NLRP3 inflammasome promotes foam cell formation in vascular smooth muscle cells and atherogenesis via HMGB1. J Am Heart Assoc 7: e008596, 2018.

34. Fan Z, Yang J, Yang J, Yang C and Guo X: HMGB1: A promising therapeutic approach for atherosclerosis. Int J Cardiol 202: 507-508, 2016.

35. Chen Z, Pan X, Sheng Z, Yan G, Chen L and Ma G: Baicalin suppresses the proliferation and migration of Ox-LDL-VSMCs in atherosclerosis through upregulating miR-126-5p. Biol Pharm Bull 42: 1517-1523, 2019.
36. Yang J, Chen L, Yang J, Ding J, Rong H, Dong W and Li X: High mobility group box-1 induces migration of vascular smooth muscle cells via TLR4-dependent PI3K/Akt pathway activation. Mol Biol Rep 39: 3361-3367, 2012.

37. Chen J, Zhang J, Xu L, Xu C, Chen S, Yang J and Jiang H: Inhibition of neointimal hyperplasia in the rat carotid artery injury model by a HMGB1 inhibitor. Atherosclerosis 224: 332-339, 2012.

This work is licensed under a Creative Commons Attribution-NonCommercial-NoDerivatives 4.0 International (CC BY-NC-ND 4.0) License. 